\title{
The roles of location specificity and masking mechanisms in the attentional blink
}

\author{
BRUNO G. BREITMEYER \\ University of Houston, Houston, Texas \\ ADDIE EHRENSTEIN and KELLY PRITCHARD \\ Rice University, Houston, Texas \\ and \\ MERRILL HISCOCK and JLL CRISAN \\ University of Houston, Houston, Texas
}

\begin{abstract}
In a series of four experiments using rapid serial visual presentations of two target letters embedded in numeral distractors, with different numbers of display positions and with or without masking, we show that (1) the nonmonotonic, U-shaped attentional blink (AB) function, which occurs when all items are presented at the same display location, is eliminated in favor of a monotonic function when targets and distractors are presented randomly dispersed over four or nine adjacent positions; (2) the $\mathrm{AB}$ monotonicity is maintained with the spatially distributed presentation even when backward masks are used in all possible stimulus positions and when the location of the next item in sequence is predictable; and (3) the U-shaped $A B$ is not due to position-specific forward or backward masking effects occurring at early levels of visual processing. We tentatively conclude that the U-shaped AB is primarily a function of the interruption of late visual processing produced when the item following the first target occurs at the same location. In order for the $\mathrm{AB}$ to severely disnupt performance, the item following the first target must be presented at the same location as the target so that it can serve both as a distractor and as a mask interrupting or interfering with subsequent visual processing.
\end{abstract}

Although the human visual system can process very large amounts of information in a single glance at the preattentive level, the information capacity of the attentive level of processing is severely limited, with estimates of the capacity of the former and latter levels at about 2,000 and 40 bits of information, respectively (Verghese $\&$ Pelli, 1992). Matters are complicated when the rate at which information is presented to the visual system is so rapid that the duration of each informative display is significantly shorter than the duration of a typical visual fixation. Even here, the visual system performs remarkably well, being able to scan rapidly presented, multielement visual displays at a rate of about 100 items/ sec when, for instance, detecting the presence of a single numeral among an array of distractor letters (Sperling, Budiansky, Spivak, \& Johnson, 1971). Consequently, it might seem that it should be easy to identify just two target letters among distractor items presented in a rapid serial visual presentation (RSVP) lasting between 1 and $2 \mathrm{sec}$ when the items are presented at a rate of about $10 \mathrm{items} / \mathrm{sec}$. However, what has now been repeatedly found is that there is a deficit in identifying the second of two targets when they

We thank Vincent Di Lollo, Thomas Ghirardelli, and Marvin Chun for their helpful comments and suggestions. Correspondence should be addressed to B. G. Breitmeyer, Department of Psychology, University of Houston, Houston, TX 77204-5341 (e-mail: brunob@uh.edu). are embedded in such a stream of distractor items (Broadbent \& Broadbent, 1987; Chun, 1997; Chun \& Potter, 1995; Raymond, Shapiro, \& Arnell, 1992, 1995; Shapiro \& Raymond, 1994; Shapiro, Raymond, \& Arnell, 1994).

This difficulty with identifying the second target (T2) has been demonstrated in a variety of tasks, ranging from the identification of two letters or words to simply detecting whether or not a black $X$ was presented in the RSVP stream at some time after the presentation of a first, more easily detectable white target (T1). The typical finding is that when $\mathrm{T} 2$ follows $\mathrm{T} 1$ by an interval ranging from about 150 to $400 \mathrm{msec}$, the accuracy of identifying T2 is significantly lower than when it follows the target immediately or after an interval longer than $400 \mathrm{msec}$ (Chun \& Potter, 1995; Raymond et al., 1992; Shapiro \& Raymond, 1994). This transient deficit in T2 identification, characterized by a U-shaped function relating the identifiability of $\mathrm{T} 2$ to its lag, or onset asynchrony (SOA), relative to $\mathrm{T} 1$, has come to be called the attentional blink (AB).

Since both T1 and T2 (as well as the distractors) are presented above threshold and identification of $\mathrm{T} 2$ is quite good when instructions to ignore T1 are followed (Raymond et al., 1992; Shapiro \& Raymond, 1994), explanations based solely on low-level, sensory limitations in detecting $\mathrm{T} 1$ are ruled out. However, the visual processing of $\mathrm{T} 1$ clearly plays a role in the occurrence and magnitude of the AB. For example, Raymond et al. (1992) 
showed that the $\mathrm{AB}$ fails to occur when no distractor item is presented immediately following $\mathrm{T} 1$. Thus, it appears that unimpeded processing of the target immediately after its offset that occurs in the immediately following blank time interval sets the stage for the successful processing of $\mathrm{T} 2$.

Accounts of the $A B$ have tended to focus on postsensory processing of T1. Raymond et al. (1995) proposed an interference model holding that the $\mathrm{AB}$ occurs after perceptual correlates of the target items have accessed visual short-term memory (VSTM). According to this account, once item information is in VSTM, target "templates" are applied, and the items that match these templates are given a high weighting for further processing. Since weightings are a limited resource, a T2 that follows too closely after $\mathrm{T} 1$ must compete with $\mathrm{T} 1$ and any distractor items for weightings. Due to this competition, T2 may receive insufficient weighting and thus fail to receive the processing required for a complete identification. Because of its temporal contiguity with $\mathrm{T} 1$, the item immediately following $\mathrm{T} 1$ (the +1 item) is assumed to gain access to VSTM at about the same time as T1. Consequently, when $\mathrm{T} 2$ immediately follows $\mathrm{T} 1$, identification of $\mathrm{T} 2$ is quite good since in this case the weightings would be shared by, rather than being competed for by, $\mathrm{T} 1$ and $\mathrm{T} 2$. Moreover, presenting a blank after the $\mathrm{T} 1$ attenuates or eliminates the $\mathrm{AB}$, since there is less information in VSTM competing for weightings. Other findings, such as that increasing the similarity between the $\mathrm{Tl}$ and the +1 item increases the $\mathrm{AB}$, also fit well with this account, since similar +1 items would be given higher weightings and thus compete more with $\mathrm{T} 2$ for further processing. Eventually, at longer target lags, recovery from the $A B$ occurs since the weights are assumed to decay with time.

A competing account for the $\mathrm{AB}$ proposed by Chun and Potter (1995) takes the processing of representations a bit further. In this so-called two-stage delay-ofprocessing account, all items are assumed to be detected and a short-lived representation of all are formed in the first stage. These representations must then be selected for further processing at the second stage. Items that are not selected by the limited capacity selection mechanism for further processing in Stage 2 are either overwritten or lost due to passive decay in the first stage. According to this account, the AB occurs when the second, selectionstage process is still busy with $\mathrm{T} 1$ when $\mathrm{T} 2$ appears. The effects of similarity on the $\mathrm{AB}$ can be explained in terms of masking: Masking of T1 can increase the time taken to complete its processing in the second stage. This, in turn, has the effect of tying up resources needed for the selection and further processing of T2, whose Stage 1 representation is decaying.

$A$ question that these accounts of the $A B$ do not fully answer is the degree to which masking effects account for the $\mathrm{AB}$. The focus of our experiments is on the role of such effects in the AB. Recently two other research groups, Seiffert and Di Lollo (1997) and Grandison, Ghirardelli, and Egeth (1997) carried out related work. Using a paradigm in which a probe $X$ presented on $50 \%$ of the trials was to be detected, Grandison et al. showed that the $A B$, which is not obtained when the +1 interval is left blank, is obtained when $\mathrm{T} 1$ is followed by a luminance mask. Since a spatially uniform luminance mask would not be expected to receive any activation for further processing, competing activation seems an unlikely candidate for the cause of the $A B$. The magnitude of the $A B$ was reduced but not eliminated when the mask was of a lower luminance. Thus Grandison et al. were able to demonstrate a direct relation between the degree to which $\mathrm{T} 1$ is masked and the magnitude of the AB. These results fit fairly well with the two-stage model, in which masking is assumed to increase the time required for the processing of $\mathrm{T} 1$.

Seiffert and Di Lollo (1997) also found support for the two-stage model in a series of experiments using a modified $\mathrm{AB}$ paradigm with the $\mathrm{X}$ probe detection task. In one of their experiments, the conventional $\mathrm{AB}$ task was compared with tasks in which the +1 item was omitted entirely or else in which the distractor item was presented simultaneously with $\mathrm{T} 1$ but the +1 interval was left blank, as in the prior condition. The $\mathrm{AB}$ was obtained in the latter as well as in the conventional condition; however, it was significantly attenuated when the +1 item was entirely omitted. Seiffert and Di Lollo reasoned, according to the interference model, that if competition for weightings in the VSTM were responsible for the AB, the magnitude of the AB would depend on the number of competing items in VSTM while the first target is being processed and the degree to which these items match the pattern template according to which weights are assigned. On the other hand, according to the two-stage model, the $\mathrm{AB}$ would be influenced by how long it takes to process $T 1$, with the time depending on the degree of masking. In another experiment, Seiffert and Di Lollo found that the $\mathrm{AB}$ was greater when a distractor, spatially displaced from the first target, was presented in the +1 order rather than simultaneously alongside the first target (with the +1 interval left blank). Seiffert and Di Lollo, in accord with Grandison et al.'s (1997) findings, reasoned that metacontrast-like masking of the first target by a spatially displaced distractor mediates the $A B$. This result supports the two-stage model rather than the interference model. According to the interference model, both the target and the simultaneously presented distractor item compete strongly for weightings in VSTM and one thus should get a strong rather than the obtained weak AB. However, according to the two-stage model, the strong metacontrast-like masking of the target by the subsequent, spatially displaced, +1 distractor would increase the time and resources required to process the target, thus delaying selection of the probe stimulus for fur- 
ther processing in the second stage while its representation in the first stage of processing is, moreover, passively decaying.

Our experiments differed from the other investigations of masking in that we used not only a conventional $A B$ paradigm in which two letters are to be detected in a stream of digit distractors (see Figure 1A) presented at the same display location, but also a modification of the paradigm that allowed for multiple display positions of the sequentially presented items (see Figure 1B).
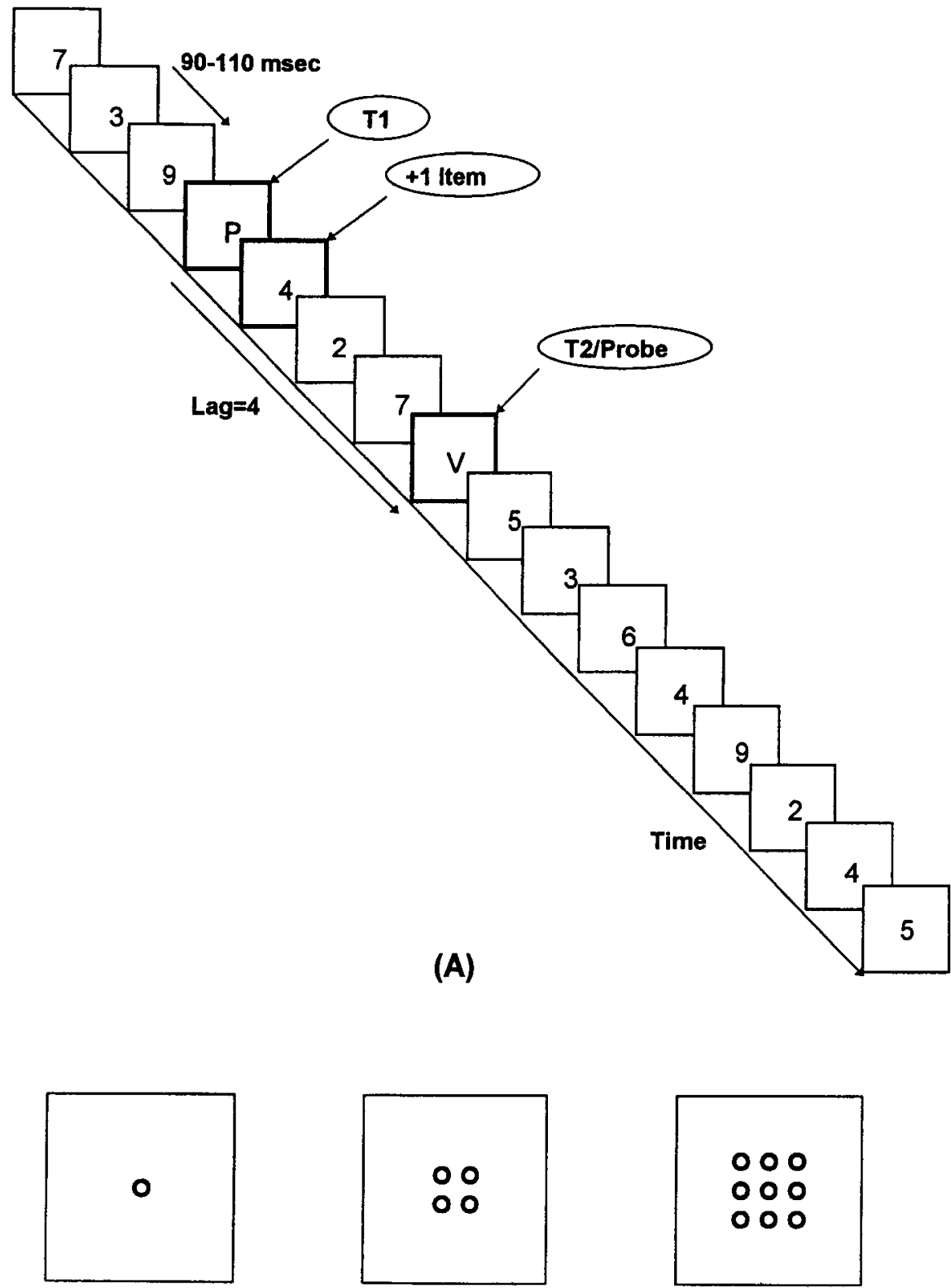

(B)

Figure 1. (A) Schematic of the rapid serial visual presentation (RSVP) of the 16 items used in the attentional blink (AB) tasks. Each item was presented for 90-110 msec and followed immediately by the next item. Target items ( $T 1$ and T2) consisted of capitalized letters; distractor items consisted of numerals. The lag between T1 and T2, shown to be 4 in the figure, could actually vary from 1 to 8. (B) Schematic illustrating how the successive RSVP items in the AB task were presented at one, four, or nine possible positions in the display. 


\section{GENERAL METHOD}

\section{Apparatus and Stimuli}

The stimuli were generated by an IBM-compatible computer controlled by Micro Experimental Laboratory software (Schneider, 1988) and displayed on a VGA 13 -in. color monitor. To minimize the problem of phosphor persistence, all items were presented as dark characters $\left(17 \mathrm{~cd} / \mathrm{m}^{2}\right)$ on a light surround $\left(107 \mathrm{~cd} / \mathrm{m}^{2}\right)$. Subjects viewed the display binocularly at a distance of $40 \mathrm{~cm}$ in a welllit laboratory room. At that viewing distance, the characters were approximately $.6^{\circ}$ high and $.5^{\circ}$ wide. All stimulus displays, which consisted of two letter targets, $\mathrm{T} 1$ and $\mathrm{T} 2$, embedded in a sequence of 14 numeral distractors, were centered on the monitor. Depending on the experiment and condition, the items, as shown in Figure 1B, were displayed in one of three spatial position formats: (1) all items presented at a single position, (2) items presented randomly at one of four positions defined by a notional $2 \times 2$ matrix (approximately $1.2^{\circ} \times 1.5^{\circ}$ ), or (3) items presented randomly at one of nine positions defined by a notional $3 \times 3$ matrix $\left(1.9^{\circ} \times 2.4^{\circ}\right)$.

\section{Procedure}

Depending on the experiment, each item was presented for $90 \mathrm{msec}$ (Experiments 1 and 4 ) or $110 \mathrm{msec}$ (Experiments 2 and 3 ) and followed immediately by the next item. In the multiple-position conditions, the sequential random positions of items were constrained so that no two temporally contiguous items were presented at the same location; that is, no display position contained two consecutive items. On any trial, T1 and T2 were drawn randomly from the capitalized letters except for I and $O$, and the distractor items were drawn randomly from the digits except for 0 and 1 . Across trials, T1 was constrained to appear randomly an equal number of times in one of the five temporal serial positions 3 through 7 , and the serial position of $\mathrm{T} 2$ relative to $T 1$, hereafter called the $T 1-T 2$ lag, was randomly varied between 1 and 8 , where a lag of 1 indicates that $\mathrm{T} 2$ immediately followed $\mathrm{T} 1$. The two serial-position randomizations were completely crossed, and this design feature was repeated six times within each spatial position condition, thus yielding $5 \times 6$ trials at each of the eight lags for a total of 240 trials per spatial format.

Subjects were informed that on each trial they would be presented with a sequence of 16 items consisting of 2 letters and 14 numbers. The subjects' task was to identify the two letters by typing them on a computer keyboard after each trial. On trials when the subject was not sure of the identity of one or the other letter, he/she was encouraged to guess. At the start of a trial, a fixation dot appeared in the center of the screen. It remained on the screen until the subject initiated a trial by pressing the space bar of the computer keyboard. After a 500-msec delay, the RSVP sequence appeared. The subject then entered his/her responses, after which the program moved on to the next trial. Although subjects were allowed to enter the two responses in either order if they were uncertain as to the presentation order of the targets, they were encouraged to attempt entering the letters in the correct order. The accuracy of the Tl and $\mathrm{T} 2$ identifications was registered and stored by the computer. Accuracies were analyzed off-line.

\section{EXPERIMENT 1}

In the first experiment, the conventional single-position format used in the study of the $\mathrm{AB}$ was compared with two other formats in which the items in the RSVP stream could be presented in one of four positions $(2 \times 2$ notional matrix) or else in one of nine positions $(3 \times 3$ notional matrix). Using the two spatially distributed display conditions was expected to reduce pattern masking due to inte- gration and interruption (Breitmeyer, 1984). However, Seiffert and Di Lollo (1997) did report a nonmonotonic $\mathrm{U}$ - or J-shaped AB function when the +1 item following $\mathrm{T} 1$ was presented in a nonoverlapping but spatially adjacent location. Such a procedure, while especially eliminating masking by integration, would still allow metacontrastlike masking obtained with spatially displaced stimuli (Breitmeyer, Battaglia, \& Weber, 1976; Breitmeyer, Love, \& Wepman, 1974) akin to the phenomenon of sequential blanking reported by several investigators (Hearty \& Mewhort, 1975; Mayzner \& Tresselt, 1970; Tresselt, Mayzner, Schoenberg, \& Waxman, 1970). On the basis of these results, we expected to obtain a strong U-shaped $A B$ with the conventional 1-position condition but also a somewhat attenuated U- or J-shaped AB with the four-position and the nine-position conditions. Moreover, since on the average the metacontrast-like masking should be stronger in the four- than in the nine-position condition due to an average smaller spatial separation between successive items in the RSVP, one might accordingly expect a stronger $\mathrm{AB}$ in the former than in the latter condition.

\section{Method}

Subjects. Eighteen subjects, 9 males and 9 females, including two of the authors (B.G.B. and M.H.), volunteered to participate in the present experiment. The remaining 16 subjects, obtained with full informed consent, were drawn from the University of Houston and Rice University undergraduate populations. All subjects had normal or corrected-to-normal vision. The ages of the subjects ranged from 19 to 52 .

Procedure. Each subject completed three blocks of 240 trials, with one block devoted to each of the three spatial formats. Order of spatial format was counterbalanced across subjects. At the start of each block, subjects performed 20 additional practice trials to familiarize them with the task. Data from these trials were not used in the statistical analysis. Subjects then performed three sets of 80 trials, with each set followed by a short rest period before proceeding. At the end of a block of trials, subjects were also given a rest period. All blocks were run in a single experimental session that lasted about $90 \mathrm{~min}$.

\section{Results and Discussion}

The data in this and all subsequent experiments consisted of T1 identification accuracy and, as in Chun and Potter (1995), of T2 identification accuracy on trials where T1 also was correctly identified, regardless of the order in which subjects identified the targets. Order of target identification was deemed unimportant because previous research has demonstrated that the order of item presentation is not preserved in the sensory buffer (Raymond et al., 1992; Weichselgartner \& Sperling, 1987).

A 3 (display position: 1,4 , and 9) $\times 8$ (lag: 1-8) repeated measures analysis of variance (ANOVA) was performed on the data. The results showed that $\mathrm{T} 1$ accuracy was affected only by display position $[F(2,34)=27.64$, $p<.0001]$. Accuracy was lower for the one-position display $(73.0 \%)$ than for the four- or nine-position displays (83.1\% and $82.0 \%$, respectively). For T2 accuracy, the results, shown in Figure 2, indicated significant main effects of display position $[F(2,34)=27.64, p<.0001]$ 


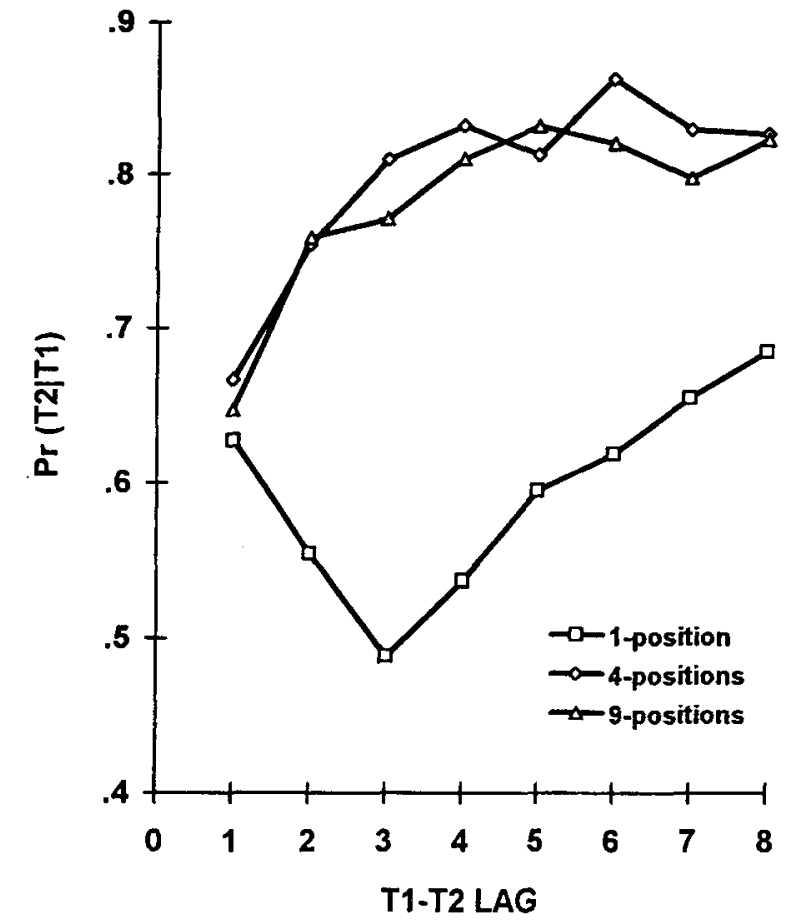

Figure 2. The proportion of correct identifications of T2 given that $T 1$ was also correctly identified as a function of the T1-T2 lag when, as indicated in the inset, the RSVP items were displayed in one, four, or nine display positions.

and T1-T2 lag $[F(7,119)=5.26, p<.0007]$ as well as a significant interaction between display position and lag $[F(14,238)=5.72, p<.0001]$. As would be expected from visual masking accounts of the $\mathrm{AB}$ (Giesbrecht \& Di Lollo, 1998); Grandison et al., 1997; Seiffert \& Di Lollo, 1997), using spatially distributed displays of four or nine positions reduced the magnitude of the $A B$. The one-position condition, as expected, resulted in the conventional nonmonotonic $U$-shaped $\mathrm{AB}$ function: $\mathrm{T} 2$ identification accuracy was $67 \%$ at a lag of 1 , dropped to a value of $50 \%$ at a lag of 3 , and then slowly increased again to a value of $68 \%$ at the highest lag of 8 . The fourand nine-position conditions, on the other hand, both yielded equal monotonic $\mathrm{AB}$ functions: $\mathrm{T} 2$ accuracy again was about $67 \%$ at the shortest lag of 1 and increased to an asymptote of about $83 \%$ at lags of 4 and higher. The results failed to confirm the expectation, based theoretically on metacontrast-like masking (Breitmeyer et al., 1976; Breitmeyer et al., 1974) and on Seiffert and Di Lollo's (1997) results, that the nine- and, particularly, the four-position displays would also produce a nonmonotonic $\mathrm{AB}$ function. The relatively high $\mathrm{T} 2 \mathrm{accu}-$ racy at lag 1 in the 1 -position condition indicates the characteristic benefit for processing the second target when it immediately follows the first. However, this benefit was no longer present in the four- and nine-position displays, in which T2 accuracy is worst when the second target immediately follows the first one. Hence, the pres- ent results indicate that in order for a $U$-shaped $A B$ function and thus a relative benefit for processing of $\mathrm{T} 2$ to occur, the T2 must not only follow T1 immediately in time but also be presented in the same spatial location. If that is so, $\mathrm{T} 2$ is a +1 item that serves not only as a target presented at the same spatial location as $\mathrm{T} 1$ but also as a spatially overlapping backward mask of T1. Hence, it is not clear whether the location specificity pertains to T2's role as a target or as a backward mask. The following experiment addresses the latter role by comparing $\mathrm{AB}$ functions obtained with the +1 items either containing or not containing a spatially overlapping mask of $\mathrm{T} 1$.

\section{EXPERIMENT 2}

If the additional crucial factor for obtaining a nonmonotonic $A B$ function is that the +1 item falls in the same spatial location as $\mathrm{T} 1$ and thus acts as a spatially overlapping pattern mask, one should be able to obtain a nonmonotonic $\mathrm{AB}$ function not only in the one-position display but also in the four- and nine-position displays provided that each item in the RSVP stream is also masked by an immediately following and spatially overlapping pattern. On the basis of a related rationale used to measure "attentional dwell time" in human vision, Duncan, Ward, and Shapiro (1994) used a display in which T1 and T2 (1) were each followed only by a single overlapping pattern mask and (2) were presented in nonoverlapping display locations at variable T1-T2 SOAs. Analogous to the conventional U-shaped AB effect, Duncan et al.'s results showed that $T 2$ recognition was a $J$-shaped function of positive T1-T2 SOA. These results would lead one to expect to find similar results in an AB task in which items, no two successive items of which are presented in the same display position, are each followed by a spatially overlapping mask. To test this hypothesis, we again employed the unmasked four- and nine-position conditions, as in Experiment 1. However, the additional crucial conditions in the present experiment, as illustrated in Figure 3, entailed presentation of a mask following each stimulus. Temporally contiguous items were again presented in different spatial locations, but the immediately following item was accompanied by a simultaneously presented "\&" character that fell in the same spatial location as the preceding item. Thus the $\&$ character served as a spatially overlapping pattern mask for each of the target and distractor items in the RSVP. If the crucial role of the +1 item is to serve as a backward mask of T1, two main results should obtain: (1) T2 accuracy overall should be higher in the unmasked than in the masked condition, and (2) a $\mathrm{U}$ - or J-shaped $\mathrm{AB}$ effect should be obtained in the masked but not in the unmasked condition.

\section{Method}

Subjects. Sixteen subjects, 8 males and 8 females, including two of the authors (B.G.B. and J.C.) volunteered to participate in the present experiment. The remaining 14 subjects, obtained with full informed consent, were drawn from the University of Houston and 


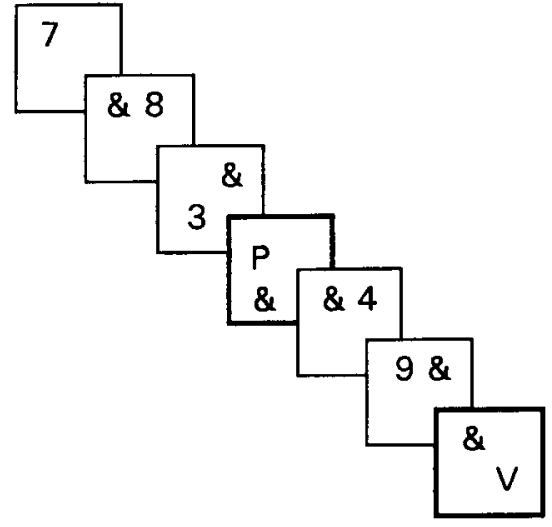

Figure 3. Schematic representation of successive and spatially nonoverlapping items each of which was accompanied by an ampersand serving as a spatially overlapping pattern mask of the just-prior item.

Rice University undergraduate populations. All subjects had normal or corrected-to-normal vision. The ages of the subjects ranged from 19 to 50 .

Procedure. Each subject completed four blocks of 240 trials, with one block devoted to each of the two spatial formats (four- and nine-position) with and without masking. Order of blocks was counterbalanced across subjects. At the start of each block, subjects performed 20 additional practice trials to familiarize them with the task. Data from these trials were not used in the statistical analysis. Subjects then performed three sets of 80 trials, with each set followed by a short rest period before proceeding. At the end of a block of trials, subjects were also given a rest period. All blocks were run in a single experimental session that lasted about $120 \mathrm{~min}$.

\section{Results and Discussion}

A 2 (presentation mode: unmasked, masked) $\times 2$ (display position: 4,9$) \times 8$ (lag: $1-8$ ) repeated measures ANOVA was performed on the data. Presentation mode was the only variable to affect $\mathrm{T} 1$ accuracy $[F(1,15)=$ $38.92, p<.0001]$, with accuracy being lower in the masked $(72.4 \%)$ than in the unmasked $(87.9 \%)$ presentation. Regarding T2 performance, the results, shown in Figure 4, revealed a significant main effect of presentation mode $[F(1,15)=90.32, p<.0001]$ and of $\operatorname{lag}[F(7,105)=24.47$, $p<.0001]$. As in Experiment 1, the main effect of the four versus nine display positions was nonsignificant. In addition, all interaction effects were nonsignificant. Thus, the effect of the mask was to lower T2 accuracy uniformly by about $25 \%$ across all lags. Moreover, not only the unmasked but also the masked presentation modes produced monotonic $\mathrm{AB}$ functions. In the unmasked condition and averaged across display positions, T2 accuracy was $68 \%$ at the shortest lag of 1 and then increased to a value of about $87 \%$ at lags of 5 and greater. Similarly, in the masked condition, $\mathrm{T} 2$ accuracy was about $41 \%$ at the shortest lag and then increased to a value of about $61 \%$ at lags of 5 and longer.

The finding that masking by spatially overlapping items produced T2 accuracy deficits was expected on the basis of prior masking studies (Giesbrecht \& Di Lollo, 1998;
Grandison et al., 1997; Seiffert \& Di Lollo, 1997). However, these and related studies (Chun \& Potter, 1995; Duncan et al., 1994; Raymond et al., 1992) also showed a nonmonotonic $\mathrm{AB}$ effect with spatially overlapping pattern masking. Since the present study used spatially overlapping masks but failed to obtain such an effect, one can conclude that spatial overlap of the mask and T 1 in itself is not sufficient to produce a U- or J-shaped AB effect. An additional critical factor seems to be that the stimuli fall in the same display location. This point will be taken up again in the General Discussion section.

\section{EXPERIMENT 3}

Although the masked condition in Experiment 2 eliminated one of the differences-namely, presence and absence of spatially overlapping pattern masking - between the one-position and the four- and nine-position conditions in Experiment 1, it did not eliminate all differences. In the one-position display, not only was each item masked by the immediately following and spatially overlapping item, but also the spatial predictability or certainty of the sequence of items was much higher than in the four- and nine-position displays. In the present experiment, we investigated not only how $\mathrm{T} 2$ identification performance is affected by masking, but also how and to what extent performance depends on the level of predictability or spatial

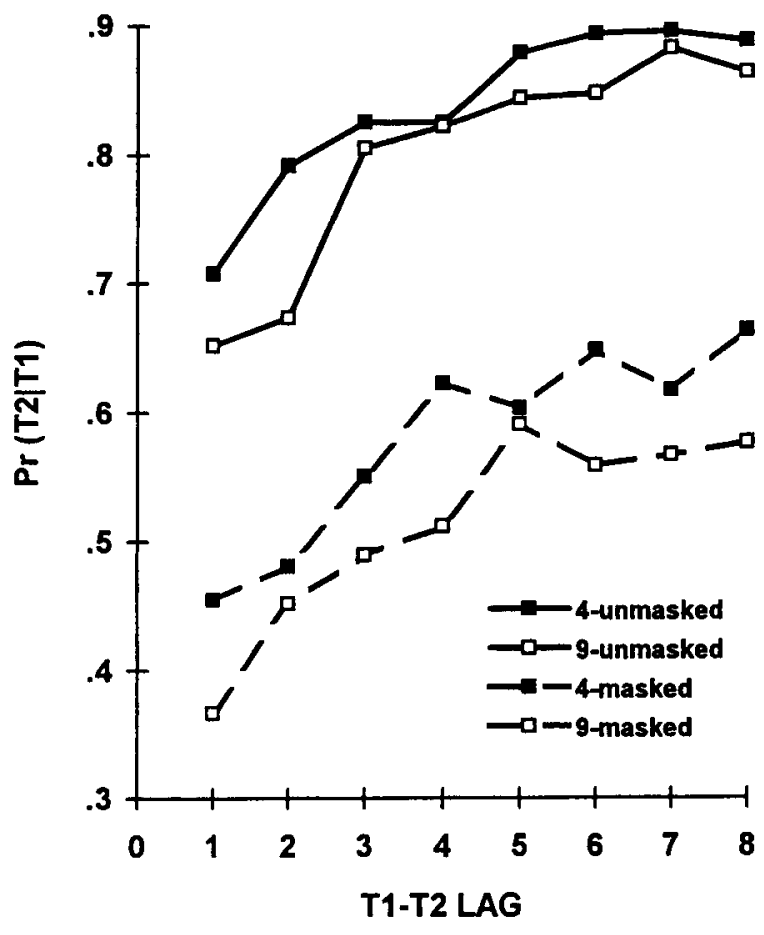

Figure 4. The proportion of correct identifications of $T 2$ given that $T 1$ was also correctly identified as a function of the T1T2 lag when, as indicated in the inset, the RSVP items were displayed in four or nine display positions and were either masked or not masked by an immediately following " $\&$ " mask. 
uncertainty of the items presented in the $\mathrm{AB}$ task. Since no differences were obtained between the four- and nineposition conditions in the prior two experiments, we employed only the four-position condition in the present experiment. We varied item-sequence predictability by presenting sequential items either randomly in the notional $2 \times 2$ display matrix, as in Experiments 1 and 2, or predictably starting in the upper left-hand corner of the matrix and advancing sequentially in a clockwise manner in subsequent presentations.

\section{Method}

Subjects. Sixteen subjects, 8 males and 8 females, including two of the authors (B.G.B. and J.C.), volunteered to participate in the present experiment. The remaining 14 subjects, obtained with full informed consent, were drawn from the University of Houston and Rice University undergraduate populations. All subjects had normal or corrected-to-normal vision. The ages of the subjects ranged from 19 to 50.

Procedure. Each subject completed four blocks of 240 trials, with each block comprising a different combination of presentation mode (masked or unmasked) and item-sequence condition (random or predictable). Order of blocks was counterbalanced across subjects. At the start of each block, subjects performed 20 additional practice trials to familiarize them with the task. Data from these trials were not used in the statistical analysis. Subjects then performed three sets of 80 trials, with each set followed by a short rest period. At the end of a block of trials, subjects were also given a rest period. All blocks were run in a single experimental session that lasted about $120 \mathrm{~min}$.

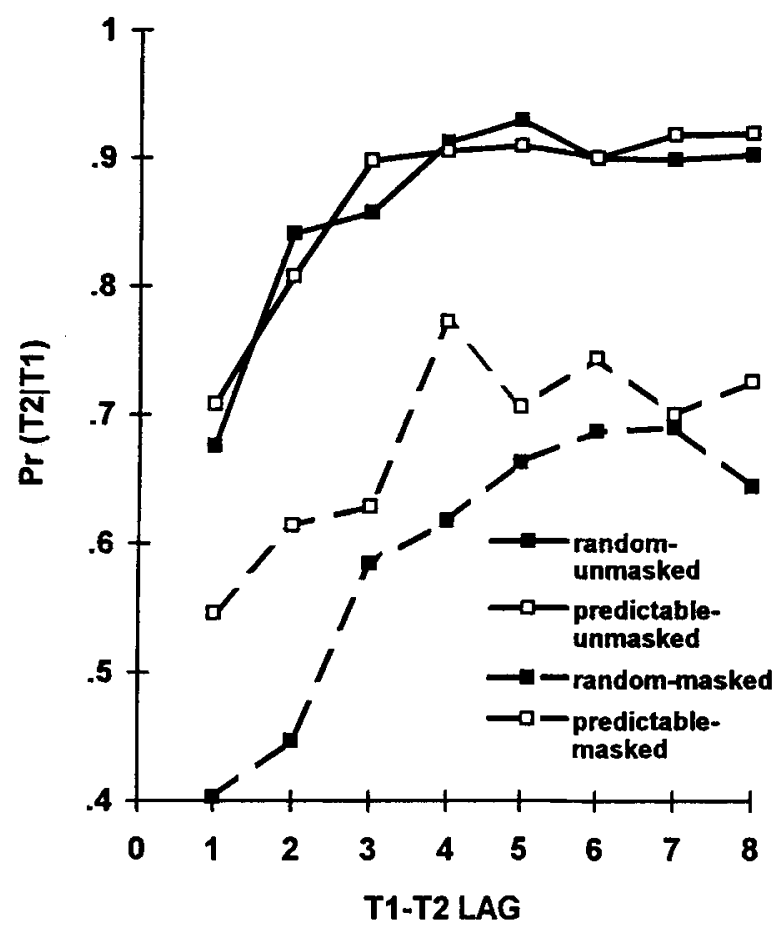

Figure 5. The proportion of correct identifications of $T 2$ given that $T 1$ was also correctly identified as a function of the $T 1-T 2$ lag when, as indicated in the inset, the RSVP items were displayed in a random or predictable spatial order and were either masked or not masked by an immediately following " $\&$ " mask.

\section{Results and Discussion}

A 2 (presentation mode: unmasked, masked) $\times 2$ (sequence: random, predictable) $\times 8$ (lag: $1-8)$ repeated measures ANOVA was performed on the data. As in Experiment 2 , the only variable to affect $T 1$ accuracy was presentation mode $[F(1,15)=26.83, p<.0001]$, with accuracy being lower in the masked $(78.8 \%)$ than in the unmasked $(93.2 \%)$ presentations. For T2 identification performance, the results, illustrated in Figure 5, show that, as in the prior experiments, there were significant main effects of presentation mode $[F(1,15)=40.42, p<$ $.0001]$ and of lag $[F(7,105)=29.04, p<.0001]$. The main effect of sequence also was significant $[F(1,15)=$ $12.70, p<.003$, indicating that predictability of the location of spatially dispersed RSVP items enhanced T2 identification performance. Of the two-way interactions, those between presentation mode and lag $[F(7,105)=$ $2.91, p<.009]$ and between presentation mode and sequence $[F(1,15)=11.94, p<.004]$ were significant; however, the interaction between sequence and lag failed to reach significance $[F(7,105)=1.18, p>.30]$. The significant presentation mode $\times$ sequence interaction reflects the fact that although the random and predictable sequences yielded almost identical performance in the unmasked presentation mode, the predictable sequence yielded noticeably better performance than the random sequence in the masked presentation mode (Figure 5). Although the three-way interaction attained significance $[F(7,105)=2.97, p<.008]$, it did not reflect a restoration of the U-shaped $\mathrm{AB}$ effect when sequence predictability was combined with masking. The failure to obtain such a $\mathrm{U}$-shaped $\mathrm{AB}$ effect is also consistent with the nonsignificant sequence $\times$ lag interaction noted above.

\section{EXPERIMENT 4}

The experiments conducted up to now have compared the conventional $\mathrm{AB}$ paradigm where all items in the RSVP stream are presented at the same display location with ones in which the items in the stream are spatially distributed. The effect of spatial distribution was to eliminate the conventional $U$-shaped $A B$ effect, even when each item was masked by a subsequent pattern (an \&) and when the locations of the items in the spatially distributed display were predictable. Even though backward masking and location predictability are inherent features of the conventional, single-location $\mathrm{AB}$ paradigm, these features alone cannot account for the U-shaped $A B$ effect. An essential feature seems to be that the items be presented at the same display location.

However, even here the types of masking affecting the $\mathrm{AB}$ must be explored more fully. As has been shown by several investigators (Chun \& Potter, 1995; Grandison et al., 1997; Raymond et al., 1992; Seiffert \& Di Lollo, 1997), when the +1 item immediately following $T 1$ is eliminated, the $A B$ is eliminated or greatly reduced. Thus, reducing or eliminating the disruptive backward masking effects of the +1 item on the processing of $\mathrm{T} 1$ 
reduces the $A B$ effect. In the case of the +1 item, backward masking can interfere with the processing of $\mathrm{T} 1$ in several ways. Backward masking, especially with spatially overlapping stimuli, can disrupt processing of the prior of the two stimuli by (1) integration (within subcortical as well cortical pathways) of the second stimulus's contrast and contour information with that of the first stimulus, (2) inhibition or suppression of pattern-analyzing channels, or (3) interruption of later, attentional processing (Breitmeyer, 1984; Michaels \& Turvey, 1979). By using a spatially displaced +1 item, as did Seiffert and $\mathrm{Di}$ Lollo (1997) or a +1 metacontrast mask surrounding but not overlapping the location of T1, as did Grandison et al. (1997), one can eliminate backward masking by integration and yet still obtain a U-shaped $A B$ function.

In the present as in the prior studies of the $A B$, the stimulus displays were viewed binocularly. With such viewing, backward masking by integration is generally weaker and extends over shorter SOAs than does forward masking by integration (Kinsbourne \& Warrington, 1962; Scharf \& Lefton, 1970; Schiller, 1966; Schiller \& Smith, 1965 ). Hence, the elimination of a relatively weak masking by integration in the backward masking case may not be very noticeable. Since forward masking by integration is generally stronger, we reasoned that the -1 item immediately preceding $T 1$ could also and more profoundly interfere by a process of integration with the processing of $\mathrm{T} 1$ and thus contribute to the presence and the strength of the U-shaped $A B$ effect. Up to now the effects of the -1 item have not been investigated. In the present experiment, we investigated the effects on the $A B$ of deleting not only the +1 item but also the -1 item. We reasoned that if the processing of $\mathrm{T} 1$ is disrupted or made more difficult by the integrative masking mechanism activated by the -1 item, then deleting the -1 item ought to eliminate or decrease noticeably the $U$-shaped $A B$ function in a way similar to what happens when one deletes the +1 item.

\section{Method}

Subjects. Sixteen subjects, 8 males and 8 females, including two of the authors (B.G.B. and J.C.) volunteered to participate in the present experiment. The remaining 14 subjects, obtained with full informed consent, were drawn from the University of Houston and Rice University undergraduate populations. All subjects had normal or corrected-to-normal vision. The ages of the subjects ranged from 19 to 50

Procedure. Each subject completed four blocks of 240 trials, with one block devoted to each of the following four presentation conditions: (1) a conventional, one-position $\mathrm{AB}$ presentation, (2) same as Condition 1 except with the +1 item deleted, (3) same as Condition 1 except with the -1 item deleted, and (4) same as Condition 1 except with both the +1 and the -1 items deleted. Order of blocks was counterbalanced across subjects. At the start of each block, subjects performed 20 additional practice trials to familiarize them with the task. Data from these trials were not used in the statistical analysis. Subjects then performed three sets of 80 trials, with each set followed by a short rest period. At the end of a block of trials, subjects were also given a rest period. All blocks were run in a single experimental session that lasted about $120 \mathrm{~min}$.

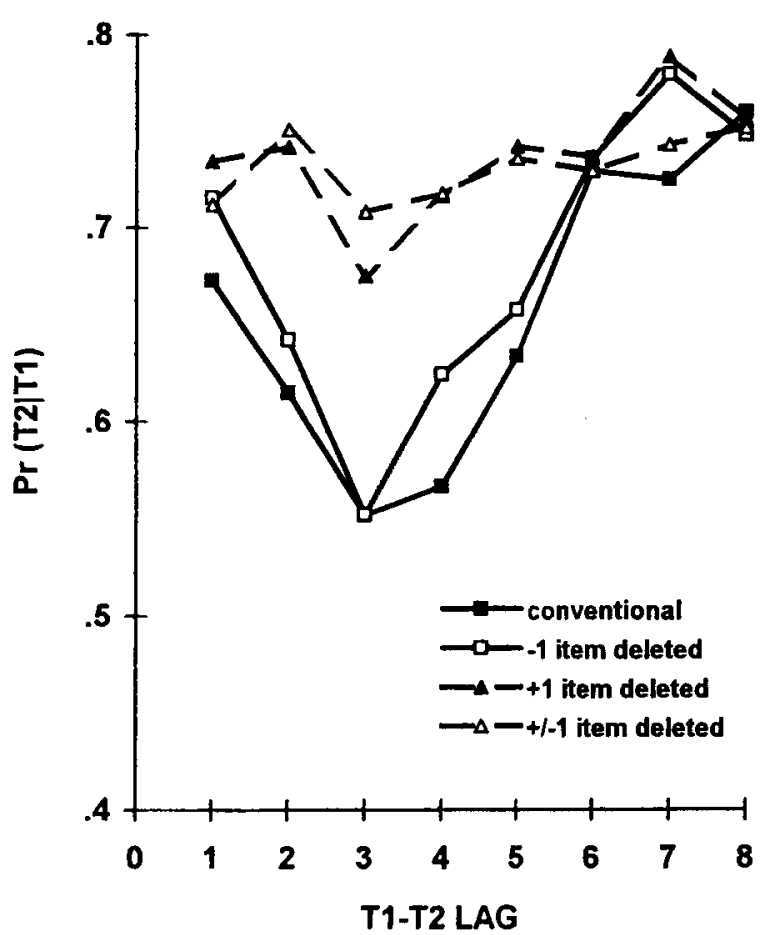

Figure 6. The proportion of correct identifications of $\mathrm{T} 2$ given that T1 was also correctly identified as a function of the T1-T2 lag when, as indicated in the inset, the RSVP items were displayed in an $A B$ task that was the conventional one, one in which the item $(+1)$ immediately following $T 1$ was deleted, one in which the item $(-1)$ immediately preceding $T 1$ was deleted, and one in which both the items $(-/+1)$ immediately preceding and following $T 1$ were deleted.

\section{Results and Discussion}

A 4 (presentation condition: conventional, +1 deleted, -1 deleted, $+1 /-1$ deleted) $\times 8$ (lag: $1-8$ ) repeated measures ANOVA was performed on the data. The only effect to reach significance for $\mathrm{T} 1$ accuracy was that of presentation condition, with accuracy being lower in the conventional and -1 deleted conditions $(79.5 \%$ and $79.6 \%$, respectively) than in the +1 and $+1 /-1$ deleted conditions $(88.4 \%$ and $89.2 \%$, respectively). Deletion of the +1 item thus improved not only T2 accuracy (Raymond et al., 1992) but also T1 accuracy. Regarding T2 performance, the results, shown in Figure 6, revealed a significant main effect of presentation condition $[F(3,45)=6.42, p<.002]$ and of lag $[F(7,105)=5.56, p<.0002]$, as well as a significant interaction $[F(21,315)=2.84, p<.0002]$. Of special interest to the present experiment are the main effect of presentation condition and the interaction effect. These significant effects are reflected, as indicated in Figure 6, by the presence of nearly equal U-shaped $A B$ effects whenever the item immediately following $\mathrm{T} 1$ is present (the conventional and the -1 deleted conditions) and the absence of any $\mathrm{AB}$ effect when the item immediately following $\mathrm{T} 1$ is deleted (the +1 and $-1 /+1$ deleted condi- 
tions). From inspection of Figure 6, it appears that T2 performance was slightly better when the -1 item was deleted than when it was not, particularly when comparing the conventional and the -1 deleted conditions. However, this apparent difference turned out to be statistically nonsignificant when submitted to a Tukey test for comparisons. The fact that performances were affected little, if at all, by deleting the -1 item immediately preceding $T 1$ indicates that any effects of forward masking by integration on the visibility of $\mathrm{T} 1$ did not significantly influence the processing of $\mathrm{T} 1$ in such a way as to impact on the subsequent identifiability of $T 2$. In other words, to obtain a $\mathrm{U}$-shaped $\mathrm{AB}$ effect, it is crucial that the immediately following $(+1)$ item exerts its masking effects on $\mathrm{T} 1$ but not that the immediately preceding $(-1)$ item does so.

\section{GENERAL DISCUSSION}

The present series of experiments has examined how location specificity and masking mechanisms associated with items in a typical RSVP task contribute to the occurrence of a nonmonotonic $\mathrm{AB}$ effect. When items are presented briefly and sequentially at the same location, a variety of masking mechanisms can be activated: forward and backward masking by integration, metacontrast masking, and backward masking by interruption of attention-demanding sequential processing (see pp. 248260 in Breitmeyer, 1984; see also Michaels and Turvey, 1979). In the following, we examine the roles of these mechanisms, relating them not only to the results of the present and prior studies but also to the contending explanatory models of the AB - the interference model of Raymond et al. (1995) and the two-stage delay-of-processing account proposed by Chun and Potter (1995).

\section{Masking by Integration}

Since $\mathrm{T} 1$ is preceded as well as followed by a distractor item in the conventional AB task, forward as well as backward masking by integration, by reducing the visibility of $\mathrm{T} 1$, can potentially interfere with its processing. According to Raymond et al.'s (1995) interference model, distractor items temporally integrated with $T 1$, that is, placed into VSTM in immediate temporal proximity with T1, ought to increase competition for weightings required for further processing of the target items. Hence deletion of the preceding, -1 item, by reducing competition for weightings, ought to produce a weaker AB effect. According to Chun and Potter's (1995) account, forward masking by integration, by degrading the quality of the $T 1$ representation in the first stage of processing, ought to increase the time required for $\mathrm{T} 1$ 's second-stage processing and thus lead to a strong $\mathrm{AB}$ effect. Hence, deleting the -1 item again should result in a weaker $A B$ effect. The results of Experiment 4, shown in Figure 6, at first glance lend some credence to either interpretive model: compared with the conventional $\mathrm{AB}$ effect, the $\mathrm{AB}$ effect obtained with the -1 item deleted appears to be slightly weaker. As noted, this difference is merely apparent, how- ever, since it was not reflected in a significant Tukey test for comparison of conditions.

However, even if this difference had been significant, a comparison of results obtained in the -1 and +1 deleted conditions demonstrates that masking by integration alone cannot be a major contributor to the $\mathrm{AB}$ effect for the following reasons. Since masking by integration is typically stronger in the forward than in the backward case (Kinsbourne \& Warrington, 1962; Scharf \& Lefton, 1970; Schiller, 1966; Schiller \& Smith, 1965), it follows that if integrative masking played a major role, then the attenuation of the $A B$ ought to be greater when the -1 item is deleted than when the +1 item is deleted. This expectation is contradicted by the clear results of Experiment 4 , showing that deleting the +1 item produces a much more dramatic attenuation of the $\mathrm{AB}$ than does deleting the -1 item. This clearly apparent difference in attenuation was reflected in a statistically significant Tukey test of comparisons. Hence, although simultaneous masking by integration, as shown by Seiffert and Di Lollo (1997), can play a strong role in producing the AB effect, both forward and backward masking by integration can play only a minor role. Additional masking mechanisms must be invoked to explain the much more critical role of the +1 item in the conventional $\mathrm{AB}$ effect. The remaining candidates are two additional backward masking mechanisms, metacontrast masking and interruption. As already amply noted by Seiffert and Di Lollo and Grandison et al. (1997), the role of these mechanisms in the AB lend support to Chun and Potter's (1995) two-stage delay-of-processing model rather than Raymond et al.'s (1995) interference model.

\section{Metacontrast and Interruption Masking}

Metacontrast masking is a type of backward masking that depends on target-mask contour interactions and is optimal at SOAs ranging from 50 to $100 \mathrm{msec}$. Although in a typical metacontrast task the target and mask contours do not overlap, metacontrast masking can also be obtained when such overlap occurs; however, when a spatially overlapping pattern mask is employed, backward masking by interruption adds to the total masking effect (Breitmeyer, 1984, pp. 131, 256-261). Since the +1 item in a conventional AB task spatially overlaps $\mathrm{T} 1$, and since the SOA separating $\mathrm{T} 1$ and the +1 item is on the order of 90-100 msec, mechanisms of metacontrast masking should be strongly activated. Moreover, at such SOAs, a subsequent mask also ought to interrupt attentiondemanding processing, in particular the read-out of items in VSTM or iconic memory (Breitmeyer, 1984, pp. 256261; Michaels \& Turvey, 1979).

Grandison et al. (1997) and Seiffert and Di Lollo (1997) confirmed the important role of metacontrast in their studies of the $A B$, in which $T 1$ was followed by either a surrounding mask stimulus or by a spatially adjacent +1 item. Both metacontrast masking conditions yielded a nonmonotonic, U-shaped $A B$ effect. When spatially adjacent stimulus items such as letters are presented in rapid 
sequence, a masking phenomenon known as sequential blanking prevails (Hearty \& Mewhort, 1975; Mayzner \& Tresselt, 1970). Breitmeyer's (1984, p. 126) analysis of sequential blanking, supported by the results of Hearty and Mewhort (1975), indicates that sequential blanking is a variant of metacontrast masking. Such sequential blanking effects also should have prevailed in the conditions of the present series of experiments in which the items were presented sequentially in adjacent spatial locations (the four-position condition of Experiment 1; the four-position unmasked condition of Experiment 2; and the random- and predictable-unmasked conditions of Experiment 3). Yet despite such metacontrast-like blanking effects, the $A B$ effect obtained in these conditions was monotonic rather than nonmonotonic.

Moreover, adding an immediately following and spatially overlapping mask to each of the items in these conditions (the four-position and nine-position masked conditions in Experiment 2; the random- and predictablemasked conditions in Experiment 3), while overall depressing the identifiability of $\mathrm{T} 2$, did not change the monotonic shape of the $\mathrm{AB}$ effect. Hence, while we agree with Grandison et al. (1997) that metacontrast masking of T 1 is sufficient to cause the $\mathrm{AB}$, neither metacontrast-like masking nor location-specific (overlap) masking by interruption is itself sufficient to explain the specifically $\mathrm{U}$-shaped $\mathrm{AB}$ effect. This conclusion holds true for the masking effects of an immediately following +1 item not only on the processing of $\mathrm{T} 1$ but also on the processing of T2. Regarding the masking effects of a following +1 item on T2, Giesbrecht and Di Lollo (1998) recently showed that the presence, as a backward mask, of a +1 item immediately following $\mathrm{T} 2$ is essential in generating a U-shaped $\mathrm{AB}$ effect. In their experiments, Giesbrecht and Di Lollo presented all items at the same display location. Thus every succeeding item served not only as a potential target for identification but also as a backward masker of the immediately prior item. In contrast, in our Experiments 2 and 3, whereas the aftercoming "\&" mask, presented along with a following item, fell at the same location as the preceding item, the following item itșelf was never presented at that location. Hence, although backward masking of T2 by the interrupting influence of the immediately following item might be necessary to obtain a U-shaped AB effect, in itself it is not sufficient. If it were, the masked conditions in our Experiments 2 and 3 also should have yielded U-shaped rather than monotonic $\mathrm{AB}$ effects. Similarly, the existence of a $\mathrm{U}$-shaped AB effect in Seiffert and Di Lollo's (1997) and Grandison et al.'s studies when metacontrast masking was employed may have been due to the fact that only the item immediately following $\mathrm{T} 1$ - the surrounding mask in Grandison et al.'s study and the +1 item in Seiffert and Di Lollo's study - were spatially displaced relative to the other items, all of which were presented at the same display location. In contrast, in the present experiments, when spatial displacement of items favoring metacontrast-like masking was employed (the unmasked conditions of Ex-

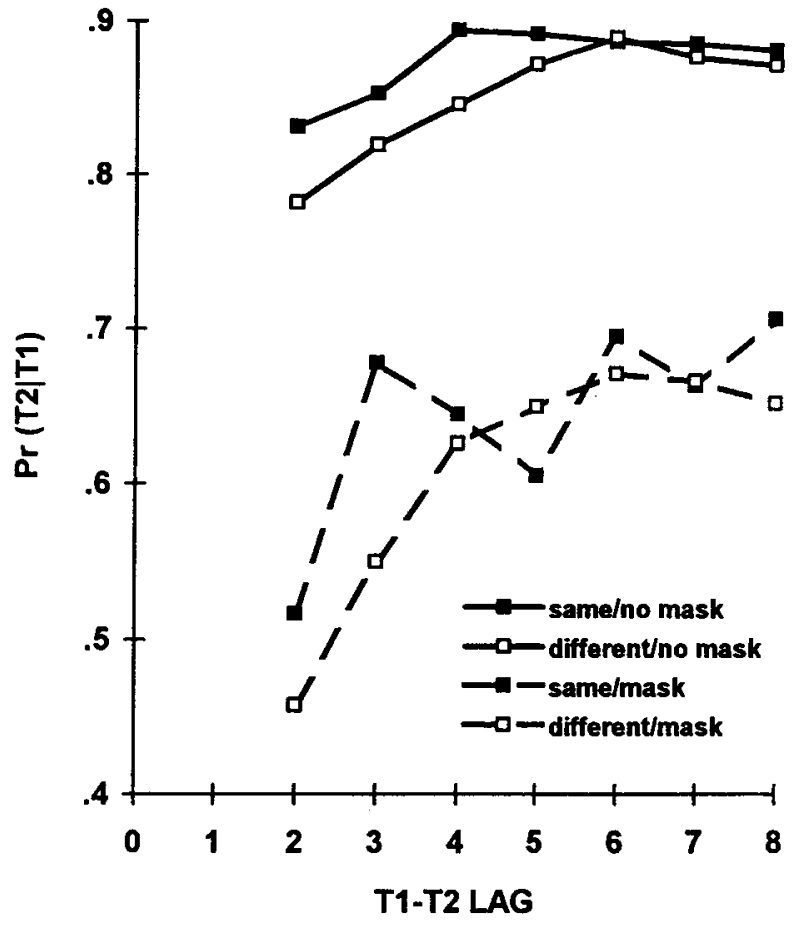

Figure 7. The proportion of correct identifications of $\mathrm{T} 2$ given that $\mathrm{T} 1$ was also correctly identified as a function of the T1-T2 lag when, as indicated in the inset, $T 1$ and $T 2$ were (1) unmasked or masked and (2) presented at the same or different display locations. Data points are based on combined results obtained from the multiple-position displays used in Experiments 1-3.

periments 2 and 3), no two sequential items were presented at the same location.

Even with this restriction, it is possible that at lag values of 2 or greater, T1 and T2 occupied the same spatial location. Thus, if $\mathrm{T} 1$, after being attentively processed at its location, is followed by intervening distractor(s) at different locations before $\mathrm{T} 2$ is presented at the same location, it is possible that inhibition of return (Lupiáñez, Milán, Tornay, Madrid, Tudela, 1997; Reuter-Lorenz, Jha, \& Rosenquist, 1996) may interfere with the discrimination and identification of $\mathrm{T} 2$ and thus contribute to the $\mathrm{AB}$ effect. Across all of the experiments, we examined the experimental conditions in which multiple positions were employed for the items in the RSVP stream. Figure 7 summarizes data from the relevant conditions in all four experiments according to whether $\mathrm{T} 1$ and $\mathrm{T} 2 \mathrm{oc}$ cupied the same display position or not as a function of masking condition and lag. As expected, the presence of a mask following the targets at their display locations decreased overall T2 identification performance. However, contrary to the inhibition-of-return hypothesis, the results indicate that when $\mathrm{T} 1$ and $\mathrm{T} 2$ occupied the same display location relative to different display locations, T2 identification actually improved rather than worsened. ${ }^{1}$ Such improvements, which can be interpreted as a facilitation of return, generally held for the unmasked as well 
as masked presentation modes and was particularly evident at the shorter T1-T2 lags corresponding to SOAs of $400 \mathrm{msec}$ and shorter. While Lupiáñez et al. (1997) also found a facilitation of return in a target discrimination task at similar cue-to-target SOAs, at larger SOAs of $700 \mathrm{msec}$ and above they obtained an inhibition of return. However, Figure 7 indicates that at lag values ranging from 6 to 8 , corresponding to SOAs ranging from about 600 to $800 \mathrm{msec}, \mathrm{T} 2$ identification performance did not depend on the location of T1. Hence, while our results indicate a facilitation of return at the shorter lag values, they fail to reveal an inhibition of return at the larger lag values.

Thus one might conclude that if the effects of metacontrast and backward masking by interruption are to express themselves in a nonmonotonic $\mathrm{AB}$ function, it is important that sequential items in an $A B$ task fall at the same display location. We draw this conclusion tentatively and with caution. The caution is necessitated not only by the results reported by Grandison et al. (1997) and Seiffert and Di Lollo (1997) but also by the results reported by Duncan et al. (1994), who, as noted in the rationale of Experiment 2, used a paradigm simpler than, but similar to, the $\mathrm{AB}$ paradigm. In their study, two targets, $\mathrm{T} 1$ and $\mathrm{T} 2$, each masked by a spatially overlapping and immediately following pattern mask, were displayed at different spatial locations and at variable T1-T2 SOAs. When identification of both targets was required, the identifiability of $T 2$ was shown to follow a $J$-shaped function for positive T1-T2 SOAs, akin to the U-shaped AB as a function of $\mathrm{T} 1-\mathrm{T} 2 \mathrm{lag}$. The $\mathrm{T} 2$ identification deficit was largest at an SOA of about $200 \mathrm{msec}$, which corresponds to an $A B$ lag of about two items given that the SOA between items is typically around $100 \mathrm{msec}$. Although the masked conditions of our Experiments 2 and 3 closely resembled the experimental procedures used by Duncan et al., we failed to obtain a U-shaped AB function under these conditions. Our procedures of course differed from those of Duncan et al. in that in ours both $\mathrm{T} 1$ and $\mathrm{T} 2$ were preceded and followed by distractor items. Thus, in addition to using backward masking effects (metacontrast and interruption) on T1 and T2, like Duncan et al., we imposed additional spatial and temporal uncertainty and thus additional processing requirements by presenting items at variable spatiotemporal locations relative to the occurrences of T1 and T2 in the RSVP. This procedural difference may also apply to comparisons of the results of our experiments with those of Experiment 3 in Grandison et al. and Experiment 4 in Seiffert and Di Lollo. In Grandison et al.'s Experiment 3, the +1 item was a white rectangle surrounding, and thus acting as a metacontrast mask of, T1. Despite the lack of spatial coincidence of the $\mathrm{T} 1$ and +1 items, a U-shaped $\mathrm{AB}$ function was obtained. Similarly, Seiffert and Di Lollo in their Experiment 4 presented the +1 item laterally displaced relative to $\mathrm{T} 1$. They too obtained a nonmonotonic $\mathrm{AB}$ function. In both of these experiments, $\mathrm{T} 1$ and $\mathrm{T} 2$ occupied the same display location since all items in the RSVP except the +1 item occupied the same display location, whereas in all of our experiments that failed to yield a $U$-shaped $\mathrm{AB}$ function, no two consecutive items were presented at the same location. Although we did not specifically explore the role of differences between the display positions of $\mathrm{T} 1$ and $\mathrm{T} 2$, on probabilistic grounds, $\mathrm{T} 1$ and $\mathrm{T} 2$ would nevertheless fall in different locations on most of the trials in the multiple-position conditions of our experiments. Since these conditions yielded monotonic instead of U-shaped AB functions, the location specificity of T1 and T2 may be a crucial factor in determining the presence or absence of a U-shaped AB function. This interpretation is consistent with recent findings that the use of different $\mathrm{T} 1$ and $\mathrm{T} 2$ display locations leads to monotonic rather than $\mathrm{U}$-shaped $\mathrm{AB}$ functions (Ghirardelli \& Egeth, 1994; Joseph, Chun, \& Nakayama, 1997). The multiple-position conditions of our experiments involved not only spatial-position uncertainty of $\mathrm{T} 1$ and $\mathrm{T} 2 \mathrm{but}$ also all of the other distractor items in the RSVP. It may be the case that spatial uncertainty eliminates a relative benefit for processing T2 when it occurs in close temporal proximity to $\mathrm{T} 1$. That the change in the shape of the $A B$ function from $U$-shaped to monotonic may be the result of eliminating a benefit for $\mathrm{T} 2$ in the +1 position is suggested by the similar performance levels at lags of 3 and higher in the single-position condition of Experiment 1 and the masked, distributed-presentation conditions of Experiment 2. However, it is also possible that requirements for attentional shifting or other mechanisms come into play in the distributed conditions. Thus, the exact mechanisms underlying the $U$-shaped $A B$ effect remain to be identified. If and how the additional displaylocation differences among RSVP items used in our experiments affect the shape of the AB function are questions we are currently investigating.

\section{REFERENCES}

BREITMEYER, B. G. (1984). Visual masking: An integrative approach. New York: Oxford University Press.

Breitmeyer, B. G., Battaglia, F., \& Weber, C. (1976). U-shaped backward contour masking during stroboscopic motion. Joturnal of $E x$ perimental Psychology: Human Perception \& Performance, 2, 167-173.

Breitmeyer, B. G., Love, R., \& Wepman, B. (1974). Contour suppression during stroboscopic motion and metacontrast. Vision Research, 14, 1451-1456.

Broadbent, D. E., \& Broadbent, M. H. P. (1987). From detection to identification: Response to multiple targets in rapid serial visual presentation. Perception \& Psychophysics, 42, 105-113.

CHun, M. W. (1997). Types and tokens in visual processing: A double dissociation between the attentional blink and repetition blindness. Journal of Experimental Psychology: Human Perception \& Performance, 23, 738-755

Chun, M. W., \& Potter, M. C. (1995). A two-stage model for multiple target detection in rapid serial visual presentation. Journal of Experimental Psychology: Human Perception \& Performance, 21, 109-127.

Duncan, J., Ward, R., \& Shapiro, K. (1994). Direct measurement of attentional dwell time in human vision. Nature, 369, 313-315.

GhIRARDElli, T. G., \& EgETh, H. E. (1994, April). What triggers an attentional blink? Paper presented at the annual meeting of the Eastern Psychological Association, Providence.

Giesbrecht, B. L., \& Di Lollo, V. (1998). Beyond attentional blinking: Visual masking by object substitution. Journal of Experimental Psychology: Human Perception \& Performance, 24, 1454-1466. 
Grandison, T. D., Ghirardelli, T. G., \& Egeth, H. E. (1997). Beyond similarity: Masking of the target is sufficient to cause the attentional blink. Perception \& Psychophysics, 59, 266-274.

HEARTY, P. J., \& MEWHORT, D. J. K. (1975). Spatial localization in sequential letter displays. Canadian Journal of Psychology, 29, 348-359.

JosePh, J. S., ChUN, M. M., \& NaKayama, K. (1997). Attentional requirements in a "preattentive" search task. Nature, 387, 805-807.

KinSbourne, M., \& WARRINGTON, E. K. (1962). Further studies on the visual masking of brief visual stimuli by a random pattern. Quarterly Journal of Experimental Psychology, 14, 235-245.

Lupiáñez, J., Milán, E. G., Tornay, F. J., Madrid, E., \& Tudela, P. (1997). Does IOR occur in discrimination tasks? Yes, it does, but later. Perception \& Psychophysics, 59, 1241-1254.

Mayzner, M. S., \& Tresselt, M. E. (1970). Visual information processing with sequential inputs: A general model for sequential blanking, displacement, and overprinting phenomena. In E. Harms \& M. E. Tresselt (Eds.), Conference on the fundamentals of psychology: Various approaches to the study of perception (Annals of the New York Academy of Science, Vol. 169, pp. 599-618). New York: New York Academy of Science.

Michaels, C. F., \& Turvey, M. T. (1979). Central sources of visual masking: Indexing structures supporting seeing at a single, brief glance. Psychological Research, 41, 1-61.

Raymond, J. E., Shapiro, K. L., \& ARnell, K. M. (1992). Temporary suppression of visual processing in an RSVP task: An attentional blink? Journal of Experimental Psychology: Human Perception \& Performance, 18, 849-860.

Raymond, J. E., Shapiro, K. L., \& Arnell, K. M. (1995). Similarity determines the attentional blink. Journal of Experimental Psychology: Human Perception \& Performance, 21, 653-662.

ReUTER-Lorenz, P. A., JHA, A. P., \& Rosenquist, J. N. (1996). What is inhibited in inhibition of return? Journal of Experimental Psychology: Human Perception \& Performance, 22, 367-378.

SCHARF, B., \& LEFTON, L. A. (1970). Backward and forward masking as a function of stimulus and task parameters. Journal of Experimental Psychology, 84, 331-338.

SCHILlER, P. H. (1966). Forward and backward masking as a function of relative overlap and intensity of test and masking stimuli. Perception \& Psychophysics, 1, 161-164.
SCHILLER, P. H., \& SMITH, M. C. (1965). A comparison of forward and backward masking. Psychonomic Science, 3, 77-78.

SCHNEIDER, W. (1988). Micro Experimental Laboratory: An integrated system for IBM PC compatibles. Behavioral Research Methods, Instrumentation, \& Computers, 20, 206-217.

SeIfFerT, A. E., \& Di Lollo, V. (1997). Low-level masking in the attentional blink. Journal of Experimental Psychology: Human Perception \& Performance, 23, 1061-1073.

ShaPIRo, K. L., \& RaYMOND, J. E. (1994). Temporal allocation of visual attention: Inhibition or interference? In D. Dagenbach \& T. H. Carr (Eds.), Inhibitory mechanisms in attention, memory and language (pp. 151-187). San Diego: Academic Press.

Shapiro, K. L., Raymond, J. E., \& ARnell, K. M. (1994). Attention to visual pattern information produces the attentional blink in rapid serial visual presentation. Journal of Experimental Psychology: Human Perception \& Performance, 20, 357-371.

Sperling, G., Budiansky, J., Spivak, J. G., \& Johnson, M. C. (1971). Extremely rapid visual search: The maximum rate of scanning letters for the presence of a numeral. Science, 174, 307-311.

Tresselt, M. E., MaYzner, M. S., Schoenberg, K. M., \& WaXman, J. (1970). A study of sequential blanking and overprinting combined. Perception \& Psychophysics, 8, 261-264.

Verghese, P., \& PELli, D. G. (1992). The information capacity of visual attention. Vision Research, 32, 983-995.

Weichselgartner, E., \& SPERLing, G. (1987). Dynamics of automatic and controlled visual attention. Science, 238, 778-780.

\section{NOTE}

1. Additional suggestive evidence for such an improvement can also be seen in the results shown in Figure 5. For the predictable-masked condition, a "spike" in T2 identification performance occurred at a lag value of 4 , consistent with the fact that at that lag value, T2 always falls in the same spatial location as $\mathrm{T} 1$.

(Manuscript received January 29, 1998; revision accepted for publication May 12, 1998.) 